\title{
Radiologic Gastrostomy: An Unmet Need!
}

\author{
Mukesh Kumar ${ }^{1}$ Anurag Chahal ${ }^{2}$
}

${ }^{1}$ Department of Radiology, BRAIRCH, All India Institute of Medical Sciences (AlIMS), New Delhi, India

${ }^{2}$ Department of Diagnostic Radiology, Mallinckrodt Institute of Radiology, Washington University in St. Louis, Missouri

Indian J Radiol Imaging 2021;31:519-520.

We recently read a manuscript, "Percutaneous gastrostomy placement by interventional radiology..." authored by Karthikumar et $\mathrm{al}^{1}$ in your esteemed journal. Authors have succinctly re-emphasized the safety and efficacy of this procedure in selected patients. Our experience with push type percutaneous radiologic gastrostomy (PRG) has also been highly rewarding and humbling. We identified a dire need for PRG in patients being denied an endoscopic gastrostomy owing to advanced cancer/stricture and embarked on a research project to kickstart these services in our tertiary care cancer hospital. During the course of this institutionally funded research, we provided free interventions to 35 such patients. The challenges we faced during this endeavor compelled us to think deeply about this facet of interventional radiology practice. We realized that most of the eligible patients are never given an option of gastrostomy. A few logistic or quasi-scientific reasons were identified as follows:

1. Lack of awareness about the exceptional safety and efficacy of the procedure leading to reduced referral rates.

2. Ease and safety of nasogastric (NG) tube placement in patients with significant dysphagia without a discussion/consideration of quality-of-life metrics.

3. Cost associated with gastrostomy may be prohibitive in some cases.

Owing to the above reasons, PRG is grossly underutilized in India. There is Level 1 evidence in literature that this intervention leads to significant improvement in quality of life and nutritional rehabilitation, essential for optimal treatment response. ${ }^{2}$ Furthermore, PRG has been found as safe as percutaneous endoscopic gastrostomy in these patients. ${ }^{3}$

Interacting with the patients during follow-up appointments gave us some perspectives. Presence of a NG tube hanging out from the nose leads to social isolation in a patient already dealing with physical stress and mental trauma of cancer. They can feel the NG tube every second for the rest
Address for correspondence Mukesh Kumar, MD, Department of Radiology, BRAIRCH, All India Institute of Medical Sciences (AIIMS), Ansari Nagar, New Delhi 110029, India (e-mail: mukeshyadav11@gmail.com).

of their life, affecting their voice, confidence, sleep and above all, acting as a continuous reminder of their cancer status.

Let us cite an example of one of our patients who was a teacher by profession. For approximately 3 months after gastrostomy tube placement, he was able to give up to 4 hours of tuition classes daily, with the $G$ tube well concealed under the clothing till it got somehow dislodged. Due to COVID-19 situation, he was unable to get his G-tube replaced and instead, got an NG tube as a rescue measure which led to self-isolation and depression leading to a rather early death. This sobbing statement from her daughter in law "Sir Agar unko wo pet wali tube dubara lag jati to wo nahi marte, wo cancer se lad lete" (sir, if he could get the G tube again, he would not have died; he would have fought the cancer) during follow-up telephonic conversation rightly emphasizes the enormous impact on the quality of life of such a patient. As interventional radiologists, we need to find solutions to increase the utilization of this valuable intervention. Our suggestions are:

1. Increasing awareness among oncology colleagues about this procedure by lectures, seminars, or informal interactions.

2. Suggesting and offering this intervention in tumor boards and multidisciplinary meetings.

3. Dispelling myths about its safety by providing data and sharing protocols from other oncology institutions around the world.

4. Substantially increasing exposure and training residents and fellows.

5. Designing frugal and indigenous gastrostomy kits to reduce cost and thus increase availability to the economically disadvantaged.

In developed countries, it is a standard of care to insert a $\mathrm{G}$ tube to supplement feeding for these patients and this is one of the most common IR procedure in oncology set-up. published online

July 27,2021
DOI https://doi.org/

$10.1055 / \mathrm{s}-0041-1733812$ ISSN 0971-3026 (c) 2021. Indian Radiological Association.

This is an open access article published by Thieme under the terms of the Creative Commons Attribution-NonDerivative-NonCommercial-License, permitting copying and reproduction so long as the original work is given appropriate credit. Contents may not be used for commercial purposes, or adapted, remixed, transformed or built upon. (https://creativecommons.org/licenses/by-nc-nd/4.0/).

Thieme Medical and Scientific Publishers Private Ltd. A-12, Second Floor, Sector -2, NOIDA -201301, India 
Although we face our unique challenges as a developing nation, this intervention has impact far and beyond nutritional supplementation and should be seen as a valuable addition in the IR practice. Instead of pointing fingers toward administration for poor resource allocation and trying to avail imported gastrostomy kits, we should take it as a collective responsibility and help in designing safe and effective indigenous kits.

In our experience, the influence of PRG on the trajectory of these patient's treatment course or nutritional rehabilitation has been immense and even in worst case scenarios, the value added to their quality of life in their last days has been very humbling.

\section{Note}

Dr. Chahal was a senior resident in the Department of Radiodiagnosis, AIIMS, New Delhi when the work was performed.

\section{Conflict of Interest}

None declared.

\section{References}

1 Karthikumar B, Keshava SN, Moses V, Chiramel GK, Ahmed M, Mammen S. Percutaneous gastrostomy placement by intervention radiology: techniques and outcome. Indian J Radiol Imaging 2018;28(2):225-231

2 Zhang Z, Zhu Y, Ling Y, Zhang L, Wan H. Comparative effects of different enteral feeding methods in head and neck cancer patients receiving radiotherapy or chemoradiotherapy: a network meta-analysis. OncoTargets Ther 2016;9:2897-2909

3 Strijbos D, Keszthelyi D, Bogie RMM, et al. A systematic review and meta-analysis on outcomes and complications of percutaneous endoscopic versus radiologic gastrostomy for enteral feeding. J Clin Gastroenterol 2018;52(9):753-764 